
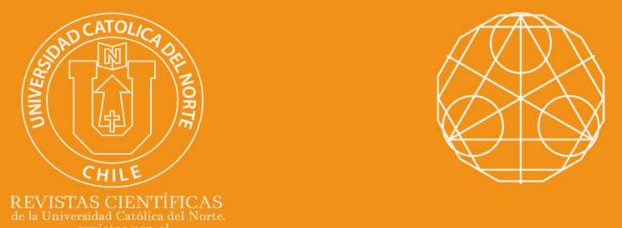

doi"10.22199/issn.0717-6279-2020-05-0077

\title{
Normed barrelled spaces
}

\section{Christopher Stuart ${ }^{1}$}

${ }^{1}$ New Mexico State University, Dept. of Mathematical Sciences, Las Cruces, NM, USA.

cstuart@nmsu.edu

\section{Abstract:}

Several conditions are given that imply barrelledness of a normed linear space. Also, examples of spaces that satisfy these properties are given.

\section{Keywords: Barrelled spaces.}

$\operatorname{MSC}(2020): 46 A 08$

\section{Cite this article as (IEEE citation style):}

C. Stuart, "Normed barrelled spaces", Proyecciones (Antofagasta On line), vol. 39, no. 5, pp. 1267-1272, Oct. 2020, doi: 10.22199/issn.0717-6279-2020-05-0077.

Article copyright: (C) 2020 Christopher Stuart. This is an open access article distributed under the terms of the Creative Commons Licence, which permits unrestricted use and distribution provided the original author and source are credited. 


\section{Introduction}

The Uniform Boundedness Principle (UBP) is one of the fundamental principles in functional analysis. The locally convex topological vector spaces for which the equicontinuity version of the UBP holds are known as barrelled spaces. See [5] for the basic results on these spaces.

To show a particular space is barrelled, a method of proof known as a gliding or sliding hump argument is frequently used. In this paper, we present a fairly general gliding hump condition on a normed linear space $X$ that implies barrelledness. We give some examples of spaces that have this condition. Ultimately, it would be highly desirable to have a gliding hump characterization of barrelled spaces.

\section{Main Results}

Definition 1. Let $X$ be a normed linear space. A bounding sequence in $X$ for an unbounded $\left(y_{n}\right) \subset X^{*}$ is a sequence $\left(x_{n}\right)$ such that $\left|y_{n}\left(x_{n}\right)\right| \rightarrow \infty$ as $n \rightarrow \infty$.

It is not difficult to show that any unbounded sequence $\left(y_{n}\right) \subset X^{*}$ has a bounding sequence $\left(x_{n}\right) \subset X$. It should be noted that each bounding sequence $\left(x_{n}\right)$ is specific to a given unbounded sequence $\left(y_{n}\right)$. In the following, $w\left(y_{n}\right)$ denotes the weak topology on $X$ induced by a sequence $\left(y_{n}\right) \subset X^{*}$. We can prove the following result.

Theorem 2. $X$ is barrelled if for every subsequence $\left(x_{n_{k}}\right)$ of a bounding sequence $\left(x_{n}\right)$ there is a further subsequence $\left(x_{n_{k_{l}}}\right)$ such that $\sum_{l=1}^{\infty} x_{n_{k_{l}}}=$ $x$, where the convergence of the series is with respect to the $w\left(y_{n}\right)$ topology on $X$.

Proof: To show barrelledness, we need to show that every pointwise bounded sequence $\left(y_{n}\right) \subset X^{*}$ is uniformly bounded on bounded sets. We do this by showing the contrapositive. We assume that $\left(y_{n}\right)$ is not uniformly bounded and show that $\left(y_{n}\right)$ is not pointwise bounded. Let $\left(x_{n}\right)$ be a bounding sequence for $\left(y_{n}\right)$.

Inductively, we can find a subsequence $\left(x_{n_{k}}\right)$ of $\left(x_{n}\right)$ such that

$$
\left|y_{n_{k}}\left(x_{n_{k}}\right)\right| \geq 2^{k}\left(M_{k}+1\right)
$$


where

$$
M_{k}=\max _{1 \leq i \leq k} \max _{1 \leq j \leq k}\left\{\left|y_{n_{i}}\left(x_{n_{j}}\right)\right|\right\}
$$

and

$$
\max _{1 \leq j \leq k}\left|y_{n_{j}}\left(x_{n_{k+1}}\right)\right| \leq 2^{-k} .
$$

The last condition is possible since $\left(x_{n_{k}}\right)$ must go to zero with respect to $w\left(y_{n}\right)$, by the assumptions in the theorem.

Note that $M_{k} \uparrow \infty$ as $k \rightarrow \infty$ since $\left(x_{n}\right)$ is a bounding sequence.

We can now find a subsequence $\left(x_{n_{k_{l}}}\right)$ such that $\sum_{l=1}^{\infty} x_{n_{k_{l}}}=x \in X$, where the convergence is with respect to the $w\left(y_{n}\right)$ topology.

Then

$$
\begin{aligned}
\left|y_{n_{k_{l}}}(x)\right| & =\left|y_{n_{k_{l}}}\left(\sum_{j=1}^{l-1} x_{n_{k_{j}}}\right)+y_{n_{k_{l}}}\left(x_{n_{k_{l}}}\right)+y_{n_{k_{l}}}\left(\sum_{j=l+1}^{\infty} x_{n_{k_{j}}}\right)\right| \\
& \geq\left|y_{n_{k_{l}}}\left(x_{n_{k_{l}}}\right)\right|-\left|y_{n_{k_{l}}}\left(\sum_{j=1}^{l-1} x_{n_{k_{j}}}\right)\right|-\left|y_{n_{k_{l}}}\left(\sum_{j=l+1}^{\infty} x_{n_{k_{j}}}\right)\right| .
\end{aligned}
$$

Using the conditions above we get

$$
\begin{aligned}
\left|y_{n_{k_{l}}}(x)\right| & \geq 2^{k_{l}}\left(M_{k_{l}}+1\right)-l M_{k_{l}}-\sum_{j=l+1}^{\infty} 2^{-k_{j}} \\
& \geq\left(2^{k_{l}}-l\right) M_{k_{l}}+2^{k_{l}}-\sum_{j=l+1}^{\infty} 2^{-k_{j}},
\end{aligned}
$$

which goes to $\infty$ as $l \rightarrow \infty$. This shows that $\left(y_{n}\right)$ is not pointwise bounded.

Sequences that have the property used in the previous proof for $\left(x_{n}\right)$ are frequently called $K$ - sequences in the literature. This name comes from the Katowice branch of the Polish Academy of Sciences, where such sequences were used to prove many classical results in functional analysis. See [1] for much more information on this subject.

As a corollary, we can give a fairly general condition for dense barrelled subspaces of $l^{1}$. In the following, $\left(e_{i}\right)$ is the sequence of canonical unit vectors in $l^{1}$ and $\Phi$ denotes $\operatorname{span}\left\{e_{i}: i \in \mathbf{N}\right\}$. It is easy to see that $\left(e_{i}\right)$ (or a subsequence thereof) forms a bounding sequence for an unbounded $\left(y_{i}\right) \subset l^{\infty}$.

Corollary 3. Let $E$ denote a subspace of $l^{1}$ that contains $\Phi$ and let $\left(t_{k}\right) \in$ $l^{1}$ be a fixed sequence with positive terms. Note that for any unbounded $\left(y_{k}\right) \subset l^{\infty}$ there is a sequence $\left(t_{k} e_{n_{k}}\right)$ for which $\sup _{k}\left|y_{k}\left(t_{k} e_{n_{k}}\right)\right|=\infty$. E is barrelled if for every sequence $\left(n_{k}\right)$ there is a subsequence $\left(n_{k_{l}}\right)$ with $\sum_{l=1}^{\infty} t_{k_{l}} e_{n_{k_{l}}} \in E$, where convergence is pointwise or coordinatewise. 
From this it is easy to show that many dense subspaces of $l^{1}$ are barrelled, including scarce copies (copies that satisfy a sparseness condition) of $l^{0}=\cap_{0<p<1} l^{p}$. This was first shown in ([2], Theorem 8). The definition of a scarce copy can be found in ([2], Section 5).

We next show that a general condition that implies the Nikodym Property (NP) for rings of subsets of a given set can be shown to be a corollary of Theorem 2. The original Nikodym Boundedness Theorem was proved for countably additive set functions defined on $\sigma$-algebras of sets. It has been generalized to finitely additive functions defined on rings of sets that satisfy various completeness conditions. The result referred to in this paper was originally proved in [4]. To show that it is a corollary to Theorem 2 we need to note that the union of a sequence $\left(E_{n}\right)$ of disjoint sets in a ring $R$ can be viewed as the summation of the characteristic functions of $E_{n} \in R$, denoted $K_{E_{n}}$. Let $S(R)=\operatorname{span}\left\{K_{E}: E \in R\right\}$. For a given $\left(y_{n}\right) \subset S(R)^{*}$, the topological dual of $S(R)$, it can be shown that any subsequence $\left(E_{n}\right)$ has a subsequence $\left(E_{n_{l}}\right)$ for which $\sum_{l=1}^{\infty} K_{E_{n_{l}}}$ converges with respect to $w\left(y_{n}\right)$, the weak topology on $S(R)$ induced by $\left(y_{n}\right)$ (See [5] Section 9.2 for details). Recall that the elements of $S(R)^{*}$ are the bounded, finitely additive measures on $R$, denoted $b a(R)$. This can be seen as a consequence of the result in ([3], IV.5.1, pp. 258-259). This result shows that the dual of $S(\Sigma)=\operatorname{span}\left\{K_{E}: E \in \Sigma\right\}$, where $\Sigma$ is an algebra of sets, is $b a(\Sigma)$. An inspection of the proof reveals that only the ring properties of $\Sigma$ are used, so the same conclusion can be be reached for $S(R)$. The Nikodym Property of $R$ is that every pointwise bounded $\left(y_{n}\right) \subset b a(R)$ is uniformly bounded. So it is equivalent to $S(R)$ being barrelled.

Definition 4. The ring $R$ is said to have the bounded subsequential completeness property $(B S C P)$ if for every unbounded $\left(y_{n}\right) \subset b a(R)$ there is a bounding sequence $\left(E_{n}\right) \subset R$ for which every subsequence $\left(E_{n_{k}}\right)$ has a further subsequence $\left(E_{n_{k_{l}}}\right)$ with $\cup_{l} E_{n_{k_{l}}} \in R$.

The following result appeared in ([4], Theorem 7). It should be noted that a modification of a theorem of Drewnowski is used in the proof. The result states that for a sequence $\left(y_{n}\right) \subset b a(R)$ and a pairwise disjoint sequence of sets $\left(E_{n}\right)$ from $R$, there is a subsequence $\left(E_{n_{k}}\right)$ such that each $\left(y_{n}\right)$ is countably additive on the ring generated by $\left(E_{n_{k}}\right)$. (See [4], Corollary $5)$. 
Theorem 5. If $R$ has BSCP then $R$ has NP.

A converse of the previous theorem can be proved in the case that $R$ is an ideal. Recall that an ideal is a ring of sets such that if $A \in R$ and $B \subset A$ then $B \in R$.

Theorem 6. If $R$ is an ideal then $R$ has $N P \Longleftrightarrow R$ has BSCP.

Proof: The sufficiency of BSCP in showing NP is stated in the previous result. Now assume that $R$ fails BSCP. Then there exists an unbounded $\left(y_{n}\right) \subset S(R)^{*}$ such that for any bounding sequence $\left(E_{n}\right)$ there is a subsequence $\left(E_{n_{k}}\right)$ for which no subsequence satisfies $\cup_{l=1}^{\infty} E_{n_{k_{l}}} \in R$. We can define $\left(z_{n}\right) \subset S(R)^{*}$ as

$$
z_{n}(A)=\left\{\begin{array}{l}
y_{n}(A) \text { for } A \subset E_{n} \\
0 \text { otherwise }
\end{array}\right.
$$

Then $z_{n}$ is unbounded on $R$ but for no $A \in R$ is $\sup _{n_{k}}\left|z_{n_{k}}(A)\right|=\infty$. This is because if there were such an $A$ then $\cup_{k=1}^{\infty} B_{n_{k}}=\cup_{k=1}^{\infty}\left(A \cap E_{n_{k}}\right) \in$ $R$ since $R$ is an ideal. But this means that $\left(B_{n_{k}}\right)$ would be a bounding sequence for $\left(z_{n_{k}}\right)$ and thus for $\left(y_{n_{k}}\right)$ which would contradict the assumption that $R$ fails BSCP.

\section{Examples}

It is shown in ([4], Proposition 10) that the ring of null sets of a strongly nonatomic submeasure $\eta$ has the BSCP. A submeasure $\eta$ on a set $S$ is strongly nonatomic if for any $\epsilon>0$ there is a finite partition of $S,\left(E_{i}\right)$, such that $\eta\left(E_{i}\right)<\varepsilon$ for all $i$.

The standard density on the natural numbers $\mathbf{N}$ is defined to be

$$
d(A)=\lim \sup _{n \rightarrow \infty} \frac{1}{n}|A \cap\{1,2, \ldots, n\}|
$$

and the uniform density on $\mathbf{N}$ is

$$
d_{U}(A)=\lim \sup _{n \rightarrow \infty} \sup _{m} \frac{1}{n}|A \cap\{m, m+1, \ldots, m+n-1\}| .
$$

It is not hard to show that these measures are strongly nonatomic on $\mathbf{N}$. Simply note that if we partition $\mathbf{N}$ into the sets $\left(E_{i}\right), 0 \leq i \leq k-1$, 
of numbers that are equal $\bmod k$, then $d\left(E_{i}\right)=d_{U}\left(E_{i}\right)=\frac{1}{k}$ for each $i$. So the rings of null sets of these measures are examples of rings that have the BSCP.

\section{References}

[1] P. Antosik and C. Swartz, Matrix methods in analysis. Berlin: Springer, 1985, doi: 10.1007/ BFb0072264

[2] G. Bennett, "Some inclusion theorems for sequence spaces", Pacific journal of mathematics, vol. 46, no. 1, pp. 17-30, 1973. [On line]. Available: https:// bit.ly/2S09iO2

[3] N. Dunford and J. T. Schwartz, Linear operators: general theory, vol. 1. New York, NY: Interscience, 1958.

[4] C.E. Stuart and P. Abraham, "Generalizations of the Nikodym boundedness and Vitali-Hahn-Saks theorems", Journal of mathematical analysis and applications, vol. 300, no. 2, pp. 351-361, Dec. 2004, doi: 10.1016/ j.jmaa.2004.06.030

[5] C. Swartz, An introduction to functional analysis. New York, NY: Dekker, 1992. 